\title{
INFORMATION DU PUBLIC
}

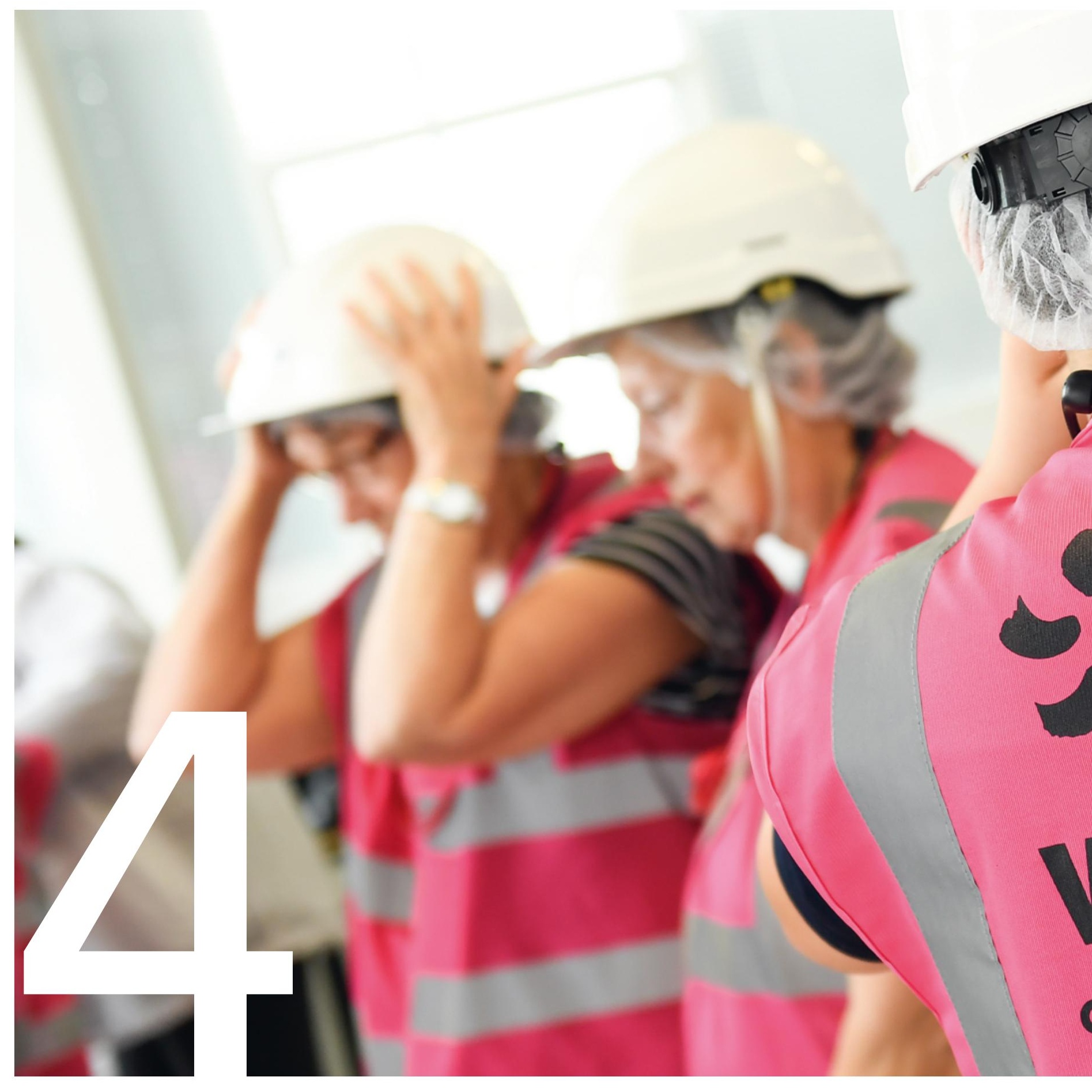




\section{Introduction}

Le public - sensible aux questions de santé et d'environnement - s'intéresse particulièrement à l'incidence des rejets des centrales nucléaires sur son milieu de vie.

Pour autant, les rejets des centrales nucléaires font peu parler d'eux dans les médias sauf à l'occasion d'incidents ou de controverses concernant leurs effets possibles sur la santé et l'environnement. Ces situations engendrent de nombreuses interrogations de la part des médias et du public auxquelles l'exploitant, l'administration et les pouvoirs publics doivent faire face.

Apporter des réponses crédibles dans ces circonstances n'est pas facile ; les sujets à traiter peuvent être complexes. Par ailleurs, pour bien comprendre l'information transmise, le public et les médias ont besoin de posséder un minimum de connaissances sur le fonctionnement des centrales nucléaires, la réglementation et de se familiariser avec un vocabulaire technique tel que dose, Sievert, Becquerel...

Pour ces raisons, l'information au public ne peut être improvisée, ni se limiter aux seules périodes de crise. Elle doit au contraire être organisée. C'est d'ailleurs l'un des objectifs de la législation en matière de transparence nucléaire qui réaffirme le droit du public à l'information et à la transparence (cf. § 2.3). Dans ce cadre, l'exploitant est tenu d'apporter régulièrement des informations sur ses activités et ceci dès la phase de conception de la centrale où des données sur les impacts prévisibles des rejets et des prélèvements d'eau doivent être présentées.

Le public est également informé par l'administration, les pouvoirs publics et les commissions locales d'information (CLI) (cf. 2.3). Pour être crédible, l'information doit s'appuyer sur des faits et des données vérifiables. Le cas échéant, il est nécessaire qu'elle apporte des « éléments de vérité scientifique » pouvant être recherchés, par exemple, auprès d'experts d'horizons différents (expertise pluraliste).

Enfin, la confiance du public dépend de la crédibilité du contrôle exercé par les autorités sur les activités de l'exploitant nucléaire. Pour cela, les autorités veillent notamment à ce que le processus conduisant à réglementer, par des décisions de l'ASN, les prélèvements d'eau et les rejets d'une centrale nucléaire prenne bien en compte l'ensemble des arguments des parties prenantes et du public en particulier (cf. chapitre 11).

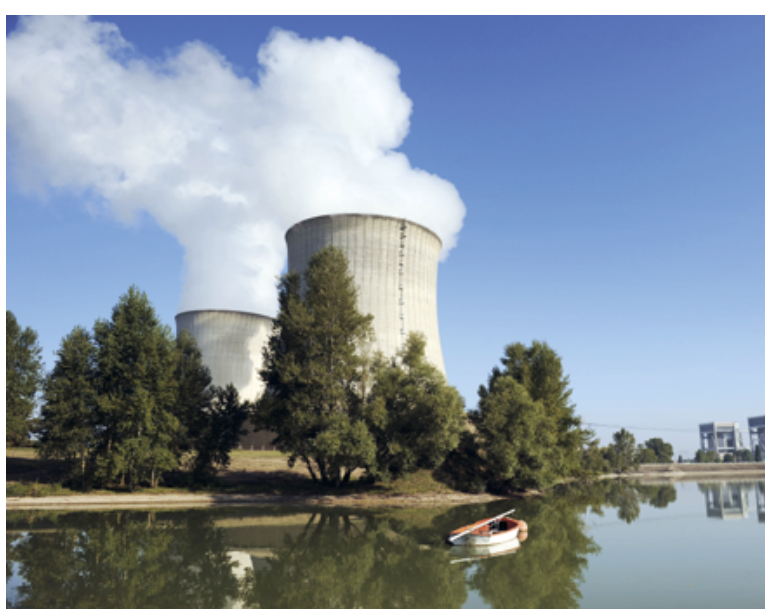

Centrale nucléaire de St-Laurent-des-Eaux sur la Loire ( $2 \times 900$ MWe en bordure de La Loire) (c) EDF - William Beaucardet

\section{Contexte législatif et réglementaire}

\subsection{AU NIVEAU INTERNATIONAL}

En matière d'environnement, les dispositions concernant l'information du public ont été confortées, entre autres, par la convention d'Aarhus' (1998). Celle-ci vise à :

- développer l'accès du public à l'information détenue par les autorités publiques ;

- favoriser la participation du public à la prise de décisions au moment de la procédure de construction d'une installation ou d'un ouvrage, lorsque toutes les options et solutions sont encore possibles et que le public peut exercer une réelle influence ;

- étendre les conditions d'accès à la justice en matière de législation environnementale et d'accès à l'information.

La convention d'Aarhus a été adoptée en droit français en 2002 (loi n² 2002-285 du 28 février 2002 puis complétée par le décret de publication du 12 septembre 2002).

1. https://www.unece.org/env/pp/introduction.html 


\subsection{AU NIVEAU EUROPÉEN}

L'Union européenne a adopté en droit communautaire la convention d'Aarhus au travers de la directive 2003/4/CE concernant l'accès du public à l'information en matière d'environnement et de la directive 2003/35/CE traitant de la participation du public aux procédures environnementales (une participation du public éclairée étant une conséquence de l'accès du public à l'information environnementale). D’après la directive 2003/4/CE, le délai de transmission des informations au demandeur ne peut excéder un mois après réception de la demande. Si la complexité ou le volume des informations le justifie, ce délai peut être porté à deux mois.

\subsection{AU NIVEAU FRANÇAIS}

\subsubsection{Disponibilité de l'information en matière nucléaire hors procédure}

\section{Affirmation du droit à l'information pour tous}

La législation' instaure un droit d'accès à l'information directement opposable à l'exploitant d'une installation nucléaire qui doit fournir à toute personne qui en fait la demande, les informations qu'il détient.

De plus, l'exploitant est tenu d'établir, sous peine de sanctions pénales, un rapport annuel public ${ }^{2}$ (cf. §3.1).

\section{Commissions locales d'information}

Les CLI ont été créées par la circulaire du 15 décembre 1981. En 2006, la législation ${ }^{3}$ a rendu obligatoire leur existence auprès de tout site nucléaire et renforcé leurs prérogatives. Composées notamment d'élus, de représentants d'associations de protection de l'environnement, et des professions médicales ${ }^{4}$, les commissions locales ont une mission de suivi, de concertation et d'information du public vivant à proximité d'un site nucléaire. Elles visent à apporter une réponse pertinente aux questions que se posent les riverains sur ces sujets.

La commission reçoit les informations nécessaires à l'accomplissement de ses missions de l'exploitant, des autorités de contrôle, des autres services de l'État, voire de laboratoires dits indépendants qu'elle a sollicités ${ }^{5}$. Elle est aussi informée, sous huit jours, de toutes les demandes formulées par le public à l'exploitant et reçoit de la même façon les réponses apportées par ce dernier ${ }^{6}$. Elle peut par ailleurs :

- faire réaliser des expertises, y compris des études épidémiologiques et faire procéder à toute mesure d'analyse de l'environnement ${ }^{7}$;

- être consultée par l'ASN et les ministres compétents sur tout projet dans le périmètre de l'INB (cette consultation est obligatoire si le projet est soumis à enquête publique) ${ }^{8}$;

- saisir l'ASN ou les ministres compétents pour toute question relevant de son domaine de compétence ${ }^{9}$;

- être saisie par le comité départemental de l'environnement et des risques sanitaires et technologiques $(\text { CODERST) })^{10}$;

- être pourvue de la personnalité juridique, avec un statut d'association et être financée, outre les subventions de l'État, par une partie de la taxe sur les $\mathrm{INB}^{11}$.

Chaque CLI assure la diffusion de l'information auprès du public visé (mairies, collèges, lycées, associations, médias, partenaires, etc.) en publiant périodiquement des bulletins, en organisant des conférences ou en renseignant son site Internet ${ }^{12}$. Les CLI sont regroupées au sein de l'Association nationale des comités et commissions locales d'information (ANCCLI).

\section{HCTISN}

La législation ${ }^{13}$ a créé, en 2006, un Haut Comité pour la transparence et l'information sur la sécurité nucléaire (HCTISN) qui peut émettre un avis sur toutes les questions relatives à l'information du public, aux risques des activités nucléaires sur la santé des personnes et sur l'environnement, et sur l'accessibilité de cette information, ainsi que proposer toute mesure de nature à garantir ou améliorer la transparence en la matière ${ }^{14}$.

\subsubsection{Disponibilité de l'information lors de procédures}

Disponibilité d'une information indépendante sur la qualité des études d'impact, des plans et projets grâce à l'Autorité environnementale

Les législations européennes et nationales (convention d'Aarhus, charte de l'environnement) prévoient que les évaluations d'impacts environnementaux des grandes opérations soient soumises à l'avis, rendu public, d'une « autorité compétente en matière d'environnement » : L'Autorité environnementale. Lorsque

\footnotetext{
1. Articles L. 125-10 à L. 125-40 du Code de l'environnement.

2. Articles L. 125-15 à L. 125-16 du Code de l'environnement.

5. Article L. 125-24 du Code de l'environnement.

6. Article L. 125-25 du Code de l'environnement.

7. Article L. 125-24 du Code de l'environnement.

8. Article L. 125-26 du Code de l'environnement.

9. Article L. 125-27 du Code de l'environnement.

10. Article L. 125-28 du Code de l'environnement.

11. Article L. 125-31 du Code de l'environnement.

12. Article L. 125-17 du Code de l'environnement.

13. Articles L. 125-34 à L. 125-40 du Code de l'environnement.

14. Article L. 125-34 du Code de l'environnement.
}

3. Articles L. 125-17 à L. 125-33 du Code de l'environnement, article R. 125-50 et suivants du Code de l'environnement.

4. Liste complète de composition des CLI à l'article L. 125-20 du Code de l'environnement. 
la saisine de l'Autorité environnementale est relative à un projet, l'autorité environnementale est soit le ministre chargé de l'Environnement, soit la formation d'autorité environnementale du CGEDD ${ }^{1}$ ou, localement, les missions régionales d'autorité environnementales du CGEDD².

Lorsque cette saisine vise les plans et programmes, elle peut être assurée par la formation d'autorité environnementale du CGEDD ou sa mission régionale d'autorité environnementale ${ }^{3}$.

\section{Disponibilité de l'information lors de l'enquête publique}

Le public est informé d'un nouveau projet ou d'une modification d'une installation nucléaire de base soumise à évaluation environnementale (par exemple : certaines modifications notables soumises à autorisation, les modifications substantielles ainsi que certaines procédures associées à la phase de démantèlement) par l'intermédiaire de l'enquête publique prévue par la réglementation ${ }^{4}$. Cette dernière donne la possibilité de prendre connaissance du projet et de formuler des observations qui devront être prises en considération par le porteur de projet.

En complément, et en vue d'assurer une information aussi complète que possible lors de l'élaboration de grands projets, une procédure d'information et de participation du public est susceptible d'être assurée sous l'égide de la Commission nationale du débat public (CNDP). Cette autorité administrative indépen- dante détermine - au regard de critères légaux - si le Projet doit notamment faire l'objet d'un débat public ou d'une concertation préalable.

\section{Disponibilité de l'information dans le cadre de la mise à la disposition du public par voie électronique mise en œuvre par l'ASN}

Le Code de l'environnement prévoit la mise à la disposition du public par voie électronique des décisions d'autorités publiques susceptibles d'avoir une incidence directe et significative sur l'environnement ${ }^{5}$.

Sur la base de ces principes généraux, L'ASN peut décider de mettre à disposition du public par voie électronique, sur son site Internet, ses projets de décisions réglementaires à caractère technique et ses décisions individuelles. Lors de la seconde hypothèse, si le projet de décision découle d'une demande de l'exploitant, alors le dossier de demande est également mis à disposition du public par voie électronique. Lorsque le volume ou les caractéristiques du projet de décision ou du dossier de demande ne permettent pas sa mise à disposition par voie électronique, le public est informé, par voie électronique, de l'objet de la procédure de participation et des lieux et horaires où l'intégralité du projet ou du dossier de demande peut être consultée.

Au plus tard à la date de la mise à disposition ou de l'information prévue à l'alinéa précédent, le public est informé, par voie électronique, des modalités de la procédure de participation retenues.

\section{Information du public par l'exploitant}

" Dire au public tout ce qu'il n'aimerait pas apprendre par d'autres que nous » peut résumer la doctrine d'EDF en matière de transparence.

Pour rester crédible et légitime à poursuivre ses activités, l'exploitant ne peut agir dans l'ombre, ni cacher les difficultés techniques auxquelles il peut être confronté.

Ceci répond également à une attente sociétale de plus en plus forte qui se manifeste notamment à l'occasion des demandes d'autorisation (enquête publique, mise à disposition du public, débat public, concertation préalable) et des réunions des CLI. Dans ces circonstances, l'exploitant ne peut pas simplement signaler qu'il respecte la réglementation. Il se doit d'expliquer les actions qu'il met en œuvre pour éviter, et réduire - autant que raisonnablement possible - les rejets et les impacts de ses installations et montrer comment il tient compte des demandes du public et des parties prenantes.

Les moyens par lesquels l'exploitant informe le public sont très variés (bulletins d'informations et rapports

1. Conseil général de l'environnement et du développement durable.

2. Article R. 122-6 du Code de l'environnement.

3. Article R. 122-17 du Code de l'environnement.

4. Confer en ce sens les dispositions communes à tous les projets (articles R. 123-1 à R.123-46), et les dispositions complémentaires spécifiques au régime INB (articles R. 593-5, R. 593-21 à R. 593-24 et R. 593-57 du Code de l'environnement.

5. Articles L. 123-19-1 et L. 123-19-2 du Code de l'environnement. 
d'activité transmis à la CLI, site Internet, organisation de visites des installations...). En cas d'événement anormal à l'origine de rejets incontrôlés, l'exploitant en informe rapidement les autorités, les pouvoirs publics et les médias. Dans ces situations, rapidité, fiabilité et sincérité du message sont des éléments essentiels pour éviter la propagation de fausses informations auprès du public.

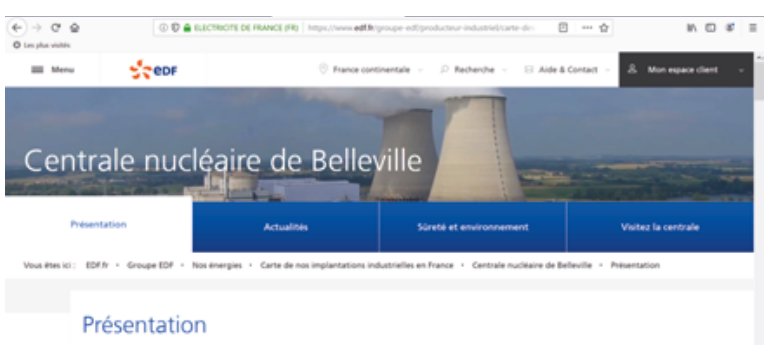

Site Internet du CNPE de Belleville-sur-Loire https://www.edffr/groupe-edf/producteur-industriel/ carte-des-implantations/centrale-nucleaire-de-belleville/presentation

\subsection{RAPPORTS À FOURNIR AU TITRE DE LA TRANSPARENCE EN MATIĖRE NUCLÉAIRE}

Au titre de la législation sur la transparence en matière nucléaire, l'exploitant est tenu d'établir un rapport annuel', rendu public, couvrant un champ d'informations très large puisqu'il doit aborder, en plus des questions d'environnement, les dispositions prises en matière de sûreté nucléaire et de radioprotection. Ce rapport est soumis au comité social et économique (remplaçant le CHSCT) de la centrale nucléaire qui peut formuler des recommandations ${ }^{2}$. Celles-ci sont annexées au rapport qui doit être transmis, avant le 30 juin de l'année suivante à l'ASN ${ }^{3}$, à la CLI et au HCTISN 4 .

\subsection{RAPPORT À FOURNIR AU TITRE DE LA RÉGLEMENTATION GÉNÉRALE APPLICABLE AUX INB}

Au titre de la réglementation générale applicable aux INB, il est demandé un rapport ciblé sur les prélèvements d'eau, les rejets d'effluents, la surveillance de l'environnement et les impacts et nuisances occasionnés durant l'année civile écoulée, ainsi que la cohérence des rejets avec les prévisions ${ }^{5}$. Ce rapport, qui peut être intégré au rapport mentionné ci-dessus est à transmettre, avant le $\mathbf{3 0}$ avril (pour certains sites) ou le 30 juin de l'année suivante, à l'ASN, la direction régionale de l'environnement, de l'aménagement et du logement (DREAL), l'agence régionale de santé (ARS), au service chargé de la police de l'eau ainsi qu'à la CLI5.

\subsection{DOCUMENTS MENSUELS « GRAND PUBLIC $~$}

Bien que les rapports annuels destinés au public soient établis par l'exploitant avec le souci de simplicité et de clarté, ces documents restent d'un abord difficile pour une personne non-initiée. Pour cette raison, l'information destinée au public est complétée par la diffusion de plaquettes ou de bulletins dits " grand public » comportant, outre la présentation synthétique des résultats, des illustrations et des photos. Ces documents de périodicité mensuelle sont diffusés aux CLI, aux associations, aux médias régionaux, aux élus locaux voire aux communes étrangères dans le cas des sites frontaliers.

\subsection{VISITE D'UNE CENTRALE NUCLÉAIRE}

Les questions que se pose le public sur le fonctionnement d'une centrale nucléaire et son impact sur l'environnement peuvent être approchées concrètement lors d'une visite de site. Chaque centrale nucléaire dispose d'un centre d'information permettant d'accueillir des personnes du public, des écoliers et étudiants pour montrer, au moyen de panneaux explicatifs, de maquettes et de films, d'une visite de terrain, la vie de la centrale et de son environnement.

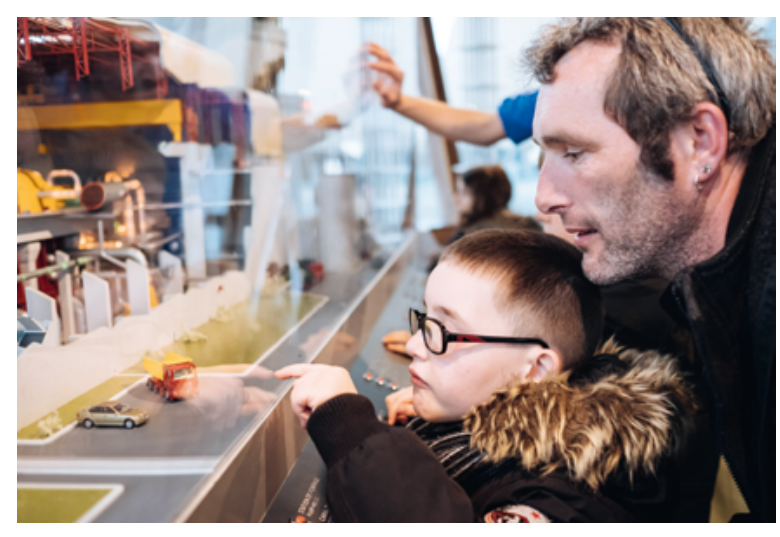

Centrale nucléaire de Belleville-sur-Loire, journée accessible à tous, 18 Mars 2017 - Enfant devant les animations au Centre d'Information du Public de Belleville-sur-Loire - ( EDF - Lewis Joly 


\subsection{PORTAIL INTERNET}

L'avènement d'Internet a permis d'élargir la diffusion de l'information à la planète tout entière. À cet égard, le site https://www.edf.fr/groupe-edf/edf-presde-chez-moi permet d'accéder à des informations actualisées sur le fonctionnement et l'environnement des centrales nucléaires d'EDF.

\subsection{COMMUNICATION SCIENTIFIQUE}

Des agents des différentes entités du groupe EDF participent à des colloques, séminaires ou encore des journées scientifiques et/ou techniques organisées au plan national ou international. Lors de ces manifestations, des communications orales et/ou affichées abordant les travaux menés par EDF, seul ou en partenariat avec d'autres organismes de recherche ou des universités, y sont présentées. Des publications scientifiques ou techniques sont également soumises à des revues avec comité de lecture afin d'y être présentées à la communauté scientifique. Certaines unités, comme EDF Lab - unité de recherche et développement du groupe EDF - organisent également des colloques ouverts au public (ex. colloques d'hydroécologie) au cours desquels des communications scientifiques sur ses travaux d'études sont exposées et les résultats publiés sur un site Internet (ex. : www. hydroecologie.org).

\section{Information du public par l'administration}

Les autorités compétentes et en particulier l'ASN mettent à la disposition du public sur Internet un grand nombre d'informations relatives à l'environnement des centrales nucléaires (les rapports annuels, les lettres de suite d'inspection, les rapports d'étude, les publications telles que la Lettre de l'ASN, les Cahiers de l'ASN, la revue Contrôle, les fiches d'information du public, les courriers de position...).

\subsection{SUR LA RADIOACTIVITÉ DE L'ENVIRONNEMENT (RNM)}

En février 2010, le Réseau national de mesure de la radioactivité de l'environnement (RNM) a été mis en ligne sur Internet. Développé sous l'égide de l'ASN en lien avec l'IRSN, ce réseau rassemble et met à la disposition du public des résultats de mesures de la radioactivité de l'environnement et des documents de synthèse sur la situation radiologique du territoire et sur l'évaluation des doses dues aux rayonnements ionisants auxquels la population est exposée (https:// www.mesure-radioactivite.fr/\#/).

Ce réseau reçoit les résultats des analyses radiologiques issues de programmes réglementaires de surveillance de l'environnement des installations nucléaires, mais également les résultats des mesures réalisées à la demande des collectivités territoriales, des services de l'État et les résultats des associations qui le sollicitent. La qualité et la fiabilité des données du réseau sont garanties par l'agrément des laboratoires pour chacun des types de mesure réalisés (cf. chapitre 10).

\subsection{SUR LES DÉBATS SCIENTIFIQUES}

Il arrive que la confiance du public soit ébranlée à la suite d'un incident, d'un accident ou d'une polémique relayée par les médias ou encore les réseaux sociaux. Dans ce cas, la simple communication sur le risque peut s'avérer insuffisante pour dissiper l'inquiétude du public. La mise en place d'une expertise dite pluraliste, faisant appel à divers acteurs (institutions, universités, associations du public, industriels...) peut s’avérer nécessaire.

\subsection{SUR LES SUJETS DE SOCIÉTÉ}

Le HCTISN peut proposer toute mesure de nature à garantir ou améliorer la transparence en la matière.

Dans le prolongement de ces missions, le HCTISN a organisé (septembre 2018 à mars 2019) une concertation sur la phase générique du $4^{\text {e }}$ réexamen périodique des réacteurs de 900 MWe du parc nucléaire français (32 réacteurs exploités par EDF et répartis sur 8 sites). Cette démarche de concertation inédite a été voulue par les membres du HCTISN afin de recueillir l'avis du public sur les conditions de poursuite du fonctionnement de ces réacteurs de 900 MWe. Cette concertation était motivée par un impératif d'intérêt général visant à :

- informer les citoyens des dispositions proposées par EDF ;

- recueillir leur avis sur ces dispositions et sur les questions à prendre en compte lors de cette première étape clé du réexamen ; 
- associer le public dès le début du processus de réexamen, avant les enquêtes publiques qui se tiendront au fil de la prochaine décennie pour chacun des réacteurs concernés.

La concertation a mobilisé des acteurs de la sûreté des centrales nucléaires: EDF, l'ASN, l'IRSN et l'ANC-
CLI. Ce dialogue avec le grand public s'est déroulé sous le regard de deux garantes de la CNDP choisies par le HCTISN. Cette démarche, forte de son succès, devrait être reconduite pour le $4^{\mathrm{e}}$ réexamen du palier $1300 \mathrm{MWe}$

\section{Information du public par les sociétés savantes}

\subsection{SOCIÉTÉ FRANÇAISE DE RADIOPROTECTION (SFRP)}

Créée en 1965, la SFRP a pour objectifs de rassembler les professionnels de la Radioprotection, de favoriser les échanges d'informations entre spécialistes et non-spécialistes, de promouvoir la culture de radioprotection et de renforcer la collaboration internationale dans ce domaine. Outre un club « jeunes sociétaires » et un « club histoire », la SFRP comprend cinq sections dont une est spécialisée sur le thème de l'environnement. Cette dernière organise régulièrement des journées techniques d'information et d'échanges sur des thématiques variées comme par exemple «Eau et radioactivité » en décembre 2014, «Évolution en matière de suivi des rejets et de surveillance de la radioactivité de l'environnement » en novembre 2015, "Air \& radioactivité » en février 2017, ou encore «Sols, sédiments et radioactivité » en février 2019.

La SFRP publie également la revue « Radioprotection » qui est à la fois une revue scientifique et un magazine professionnel.

La SFRP tient également à jour un site Internet (www. sfrp.asso.fr) compilant l'ensemble de ses activités et permettant aussi à ses adhérents de consulter l'historique des présentations réalisées lors des réunions techniques.

\subsection{SOCIÉTÉ FRANÇAISE D'ÉNERGIE NUCLÉAIRE (SFEN)}

Créée en 1973, la SFEN rassemble des ingénieurs, techniciens, chimistes, professionnels de santé, professeurs, étudiants, industriels et chercheurs du secteur nucléaire français. C'est une association qui produit et diffuse, notamment via son site Internet (www.sfen.org), des informations permettant de se familiariser avec les sciences et techniques nucléaires.

La SFEN organise également des conférences nationales et internationales comme l'« International Congress on Advances in Nuclear Power Plants", «Atoms for the Future » ou encore les «NPC Conférence ».

La SFEN publie également une revue bimestrielle intitulée «La Revue générale du nucléaire ».

\section{POUR EN SAVOIR PLUS}

- http://www.ecologie.gouv.fr/article.php3?id_article=2467(Convention Aahrus)

- www.mesure-radioactivite.fr

- www.sfrp.asso.fr

- http://europa.eu/legislation_summaries/environment/general_provisions/l28056_fr.htm (Directives européennes 2003/4/CE sur l'information du public et 2003/53 sur la participation publique)

- www.sfen.org 